\title{
Botulinum toxin injection following deep brain stimulation in generalized dystonia
}

\author{
Barbara I. Karp ${ }^{1 *}$, Vesper Fe Marie Llaneza Ramos ${ }^{2}$, Katharine Alter ${ }^{3}$ and Codrin Lungu ${ }^{4}$ \\ ${ }^{1}$ CNS, IRB, National Institutes of Health, Bethesda, MD-20892, USA \\ ${ }^{2}$ Human Motor Control Section, National Institutes of Health, Bethesda, MD-20892, USA \\ ${ }^{3}$ Functional and Applied Biomechanics Section, Rehabilitaion Medicine Department, National Institutes of Health, Bethesda, MD and Mount Washington \\ Pediatric Hospital, Washington, DC, USA \\ ${ }^{4}$ Office of the Clinical Director, National Institutes of Health, Bethesda, MD-20892, USA
}

\section{Introduction}

Deep brain stimulation (DBS) has emerged as an effective treatment for idiopathic generalized and some focal dystonias $[1,2]$. It is also increasingly utilized in patients with symptomatic or secondary dystonias, such as those due to cerebral palsy, post-traumatic dystonia, post-stroke dystonia, tardive dystonia, neurodegenerative or metabolic disorders such as pantothenate [3]. kinase-associated neurodegeneration (PKAN), and other disorders, although they may be less responsive to DBS $[1,4-8]$.

For dystonia, DBS usually targets the GPi and is often reserved for patients who are poorly controlled with oral medications and botulinum toxin (BoNT) injections. DBS typically improves generalized dystonia by about $50 \%$ on rating scales such as the Burke-Fahn-Marsden dystonia rating scale $[1,9]$.

BoNT plays a limited role in management of generalized dystonia as toxic doses would be required to treat all body areas. However, BoNT can successfully relieve spasm in discrete body areas [9-11]. Patients with generalized dystonia often receive BoNT injections for symptomatic control of specific symptoms before undergoing DBS. Since residual symptoms are present in patients with generalized dystonia following DBS, continuing BoNT injections after DBS placement may be helpful. There is, however, little literature available on the utility of botulinum toxin after DBS surgery and whether the dose, selection of muscles or response to BoNT is altered by DBS. In this small retrospective study, we evaluated the utility of BoNT following DBS surgery in patients with generalized dystonia.

\section{Methods}

The National Institute of Neurological Disorders and Stroke Human Motor Control Section clinical database was queried for patients with generalized dystonia who received BoNT injections for at least 1 year after DBS. The muscles injected, BoNT dose, and response to injection were recorded for the last injection session prior to DBS (when applicable) and followed yearly from date of DBS surgery (Table 1).

Results are described as mean (range). Response to BoNT was scored using a patient self-reported visual analog scale ranging from $0 \%$ (no improvement) to $100 \%$ (no dystonic symptoms). DBS settings were adjusted during the period of BoNT treatment by patient physicians independent of those injecting BoNT.

\section{Results}

\section{Patients}

Patient \# 1 DYT1: Patient \#1 had delayed motor milestones; she sat at 1 year of age and walked at $2 \frac{1}{2}$ years. Initial dystonic symptoms were noted at age 9 , when she developed difficulty writing, using utensils, and buttoning and "overlapping toes". No treatment was sought. Neck involvement, which was not prominent until age 19, progressed over 6 months to a fixed laterocollis. Neck pain and concerns about her appearance led to medical evaluation. The patient was of Ashkenazi Jewish descent and family history revealed a maternal aunt with generalized dystonia and a father with cervical dystonia. MRI of the brain and cervical spine was normal. Genetic testing was positive for DYT-1.

After a brief trial of levodopa without response, BoNT injections were started, targeting the neck and right hand. Injections over the next 10 years brought $50-80 \%$ improvement in neck and hand symptoms. However, the dystonia gradually worsened and spread to other body areas. Injections became less effective, yielding only about $30 \%$ benefit. She underwent bilateral globus pallidus internus (GPi) DBS surgery at age 29. BoNT injections were resumed after DBS. The injections targeted a similar number of muscles and used a dose similar to that previous to surgery. Over the next 2 years, she had a consistent response of about $40 \%$ improvement in residual dystonic symptoms with injection of 503 units (range 500 to 505) of onabotulinumtoxinA to neck muscles. She has had no adverse effects.

Patient \#2 Intrauterine Rubella: Patient \#2 had motor and cognitive development delays attributed to intrauterine rubella infection. As a child, she was able to walk and swim without difficulty. However, she gradually developed laterocollis, scoliosis, and myoclonic jerking of her neck as well as abnormal arm and leg movement and postures during her 30 s, by age 41 , she was unable to walk without assistive devices. She was unable to tolerate oral medications including trihexyphenidyl and amantadine. She received up to 200 units

Correspondence to: Barbara I. Karp, MD, National Institute of Neurological Disorders and Stroke, National Institutes of Health Bld 31, Bethesda, MD 20892 USA, Tel: 301 496-0150; Fax: 301 480-2973; E-mail: karpb@ninds.nih.gov

Key words: botulinum toxin, generalized dystonia, deep brain stimulation

Received: May 10, 2017; Accepted: May 23, 2017; Published: May 26, 2017 
Table 1. Number of muscles and doses before and annually after DBS.

\begin{tabular}{|c|c|c|c|c|}
\hline Diagnosis & Time of injection & Muscles & Total dose* & Response** $^{* *}$ \\
\hline \multirow[t]{3}{*}{ DYT1 } & Before DBS & 5 & 505 & 30 \\
\hline & $1 \mathrm{y}$ & 5 & 500 & 40 \\
\hline & $2 y$ & 5 & 505 & \\
\hline \multirow{9}{*}{$\begin{array}{l}\text { Intrauterine } \\
\text { Rubella }\end{array}$} & Before DBS & 4 & 200 & 0 \\
\hline & 0y (immediately p-dbs) & 4 & 320 & 75 \\
\hline & $1 \mathrm{y}$ & 8 & 500 & 75 \\
\hline & $2 \mathrm{y}$ & 9 & 500 & 75 \\
\hline & $3 y$ & 7 & 370 & 75 \\
\hline & $4 y$ & 6 & 360 & 75 \\
\hline & $5 y$ & 6 & 310 & 75 \\
\hline & $6 y$ & 7 & 330 & 75 \\
\hline & $7 y$ & 3 & 320 & 75 \\
\hline \multirow[t]{2}{*}{ PKAN2 } & Before DBS & 9 & 215 & 25 \\
\hline & $1 \mathrm{y}$ & Salivary glands only & & \\
\hline \multirow[t]{7}{*}{ DYT1 } & 0y (immediately p-dbs) & 8 & 200 & 15 \\
\hline & $1 \mathrm{y}$ & 9 & 660 & 20 \\
\hline & $2 y$ & 11 & 555 & 55 \\
\hline & $3 y$ & 12 & 660 & 75 \\
\hline & $4 y$ & 11 & 610 & 50 \\
\hline & $5 y$ & 13 & 595 & 60 \\
\hline & $6 y$ & 12 & 560 & 40 \\
\hline
\end{tabular}

"dose = units onabotulinumtoxinA

**Response assessed by patient/family as $\%$ improved $(0=$ none to $100=$ complete response $)$

onabotulinutoxinA to the neck and trunk without noticeable response at another institution. She underwent GPi DBS implantation at age 42. At BoNT treatment sessions over the subsequent 7 -year period with ongoing DBS, 3-9 muscles (mean $=6$ ) were injected with a mean total dose of 37 units onabotulinumtoxin A (range 310-500 units). She has had a stable response to injection, which she and her family assessed at $75 \%$ improvement. There have been no adverse effects other than temporary mild weakness in injected muscles.

Patient \#3 PKAN2: Patient \#3 had clumsiness and behavioral problems dating to early childhood. She was initially diagnosed with attention deficit/hyperactivity disorder and treated with antidepressants and stimulants, which elicited vocal and motor tics. During childhood and adolescence, she also received risperidone and topiramate for behavioral symptoms. When writing, she had a tight grip on the pen and switched from writing with her right hand to the left hand in $7^{\text {th }}$ grade. Slurred speech, involuntary jaw opening and dystonic posturing of her arm and leg were first noticed at age 16. Dystonia gradually worsened to involve all extremities, the trunk and the face and jaw. CT scan of the brain showed basal ganglia calcification. Genetic testing was positive for PKAN 2. Levodopa was tried without response. She began BoNT injections for hand dystonia with moderate response at another institution according to prior physician notes. When first seen at NIH, her main impairment from the generalized dystonia included slurred speech, jaw opening dystonia, hypersalivation, and difficulty walking. Injections at NIH with up to 440 units onabotulinumtoxin A before DBS brought about $10 \%$ improvement. She underwent GPi DBS at age 25. Following DBS, she only had a onetime injection of 10 units to each lateral pterygoid for her dystonia. She discontinued limb and neck injections because of remarkable improvement from DBS. However, she elected to continue BoNT injections for sialorrhea only with mean 80 units (range 60 to 100) to each parotid, and 32 units (range 15 to 60) to each submandibular gland. Switching to rimabotulinumtoxinB did not result in further improvement.

Patient \#4 DYT1: Patient \#4, a Guatemalan woman of Chinese ancestry, with otherwise normal birth and delivery and early childhood milestones, was diagnosed as having a psychogenic movement disorder when she developed abnormal hand and feet posturing at age 10. Symptoms worsened despite psychiatric therapy and neurologic therapy, including trial of benzodiazepines, antidepressants, levodopa, baclofen and trihexiphenidyl, such that by age 24 , dystonia had generalized to involve the trunk and all extremities and she required a wheelchair. Genetic testing was positive for DYT1. She had GPi DBS initiated at age 30. BoNT injections were first started after DBS to address residual dystonic symptoms. Over the next 6 years, she received a mean dose 627units (range 200-785) with a stable response rated 50 $\pm 8 \%$ improvement. She has reported no adverse effects of injection.

\section{Discussion}

While the first line treatment for focal dystonia, botulinum toxin injection is not uncommonly used in patients with generalized dystonia to address focally disabling symptoms. GPi DBS, however, is a better approach for these patients, reducing generalized dystonic symptoms by $50-80 \%$ and improving quality of life, comfort and function $[1,9]$. Although patients with generalized dystonia that is acquired or due to a degenerative underlying disease have a less robust response to DBS, they often benefit sufficiently to justify the risks of surgery [7]. DBS is increasingly being used in this population. Both BoNT injections and DBS offer the advantage of being safe to use in combination with other therapeutic modalities, including each other. The lack of published literature on the utility of botulinum toxin injection after DBS implantation leaves little to guide physicians on their combined use.

This report of 4 cases, 2 patients with DYT-1 dystonia, 1 with acquired dystonia from perinatal infection and 1with dystonia due to the neurodegenerative disease PKAN, provides the first information on the utility of BoNT in combination with DBS. Although we have followed only a small number of patients receiving BoNT after DBS for generalized dystonia, their cases are informative. These patients show that botulinum toxin can continue to be effective in managing residual dystonic symptoms over up to at least 7 years and that the dose and response can remain stable with DBS, including stimulator setting adjustments. Further study is needed to better delineate the role for BoNT in management of dystonia in combination with DBS.

\section{Acknowledgment}

The authors would like to thank Elaine Considine, R.N. for her help with coordinating the elements of the manuscript.

The authors acknowledge that this manuscript was prepared as part of their official duties as employees of the Department of Health and Human Services (DHHS). Work presented here does not officially express the opinion of DHHS.

This work was supported in part by a training grant to Dr. Ramosfrom the Dystonia Medical Research Foundation. This research was supported by the Intramural Research Program of the NINDS, NIH.

\section{References}

1. Loher TJ, Capelle HH, Kaelin-Lang A (2008) Deep brain stimulation for dystonia: outcome at long-term follow-up. Journal of neurology 255: 881-884.

2. Moro E, Gross RE, Krauss JK (2013) What's new in surgical treatment for dystonia? Movement disorders. Journal of the Movement Disorder Society 28: 1013-1020.

3. Eltahawy HA, Saint-Cyr J, Giladi N, Lang AE, Lozano AM (2004) Primary dystonia is more responsive than secondary dystonia to pallidal interventions: outcome after pallidotomy or pallidal deep brain stimulation. Neurosurgery 54: 613-619.[Crossref] 
4. Krause M, Fogel W, Tronnier V (2006) Long-term benefit to pallidal deep brain stimulation in a case of dystonia secondary to pantothenate kinase-associated neurodegeneration. Movement disorders. Journal of the Movement Disorder Society 21: 2255-2257.

5. Hedera P (2014) Treatment of Wilson's disease motor complications with deep brain stimulation. Ann N Y Acad Sci 1315: 16-23.[Crossref]

6. Olaya JE, Christian E, Ferman D (2013) Deep brain stimulation in children and young adults with secondary dystonia: The Children's Hospital Los Angeles experience. Neurosurgical focus 35: E7.

7. Castelnau P, Cif L, Valente EM (2005) Pallidal stimulation improves pantothenate kinase-associated neurodegeneration. Annals of neurology 57: 738-741.
8. Zhang JG, Zhang K, Wang ZC, Ge M, Ma Y (2006) Deep brain stimulation in the treatment of secondary dystonia. Chin Med J (Engl) 119: 2069-2074.

9. Vidailhet M, Vercueil L, Houeto JL (2007) Bilateral, pallidal, deep-brain stimulation in primary generalised dystonia: a prospective 3-year follow-up study. Lancet neurology 6: $223-229$.

10. Albanese A, Asmus F, Bhatia KP (2011) EFNS guidelines on diagnosis and treatment of primary dystonias. Eur J Neurol 18: 5-18.

11. Simpson DM, Blitzer A, Brashear A (2008) Assessment: Botulinum neurotoxin for the treatment of movement disorders (an evidence-based review): report of the Therapeutics and Technology Assessment Subcommittee of the American Academy of Neurology. Neurology 70: 1699-1706.

Copyright: (C2017 Karp BI. This is an open-access article distributed under the terms of the Creative Commons Attribution License, which permits unrestricted use, distribution, and reproduction in any medium, provided the original author and source are credited. 